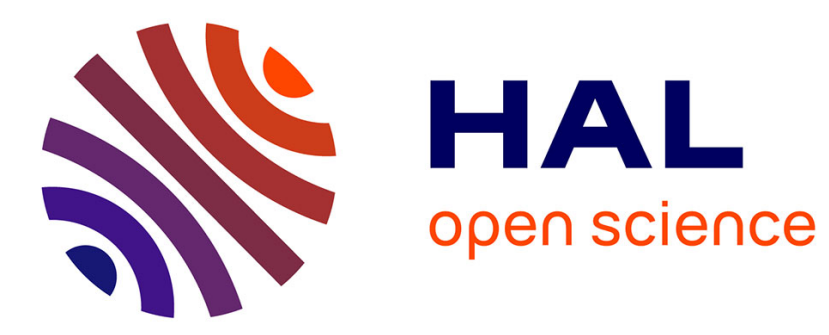

\title{
Whole scalp EEG power change is not a prerequisite for further EEG processing
}

Dirk de Ridder, Marco Congedo, Jae-Jin Song, Sven Vanneste

\section{To cite this version:}

Dirk de Ridder, Marco Congedo, Jae-Jin Song, Sven Vanneste. Whole scalp EEG power change is not a prerequisite for further EEG processing. Hearing Research, 2016, 339, pp.215-216. hal-01370794

\section{HAL Id: hal-01370794 \\ https://hal.science/hal-01370794}

Submitted on 23 Sep 2016

HAL is a multi-disciplinary open access archive for the deposit and dissemination of scientific research documents, whether they are published or not. The documents may come from teaching and research institutions in France or abroad, or from public or private research centers.
L'archive ouverte pluridisciplinaire HAL, est destinée au dépôt et à la diffusion de documents scientifiques de niveau recherche, publiés ou non, émanant des établissements d'enseignement et de recherche français ou étrangers, des laboratoires publics ou privés. 
Letter to the Editor published on Hearing research (Elsevier), 339 (2016), 215-216

\title{
Whole scalp EEG power change is not a prerequisite for further EEG processing
}

\author{
Dirk De Ridder*1, Marco Congedo 2, Jae-Jin Song 3, Sven Vanneste 4
}

\begin{abstract}
1 Department of Surgical Sciences, Section of Neurosurgery, Dunedin School of Medicine, University of Otago, Dunedin, New Zealand
2 GIPSA-lab, CNRS, Grenoble University, Grenoble Institute of Technology, Grenoble, France

3 Department of Otorhinolaryngology Head-and-Neck Surgery, Seoul National University Bundang Hospital, Seongnam, South Korea

4 School of Behavioral and Brain Sciences, The University of Texas at Dallas, USA

* Corresponding author. Brai2n, Department of Surgical Sciences, Section of Neurosurgery, Dunedin School of Medicine, University of Otago, Dunedin, New Zealand. E-mail address: dirk.deridder@otago.ac.nz (D. De Ridder).
\end{abstract}

\section{Dear editor,}

with interest we read the article "Whole scalp resting state EEG of oscillatory brain activity shows no parametric relationship with psychoacoustic and psychosocial assessment of tinnitus: A repeated measures study" of Pierzycki and coworkers. The authors performed a test-retest EEG study and found no correlation between psychoacoustic and psychosocial assessments and whole scalp EEG power. The authors wrote, "We did use spatial analysis to attenuate artifacts but not a spatial analysis with a source model. Our reason for not doing so was that there were no trends in our results to suggest that spatial analysis would be effective and so post-hoc data exploration cannot be justified. Only if general effects are found at the whole scalp level would it be appropriate to undertake further refined spatial analysis" (Pierzycki et al., 2015). This last sentence is especially remarkable for at least 6 reasons pertaining to: philosophical, historical, pathophysiological, clinical, network science, and statistical points of view. Moreover the authors conclude, "... that whole scalp-EEG oscillatory brain activity should not be considered as a viable biomarker or outcome measure in clinical trials of tinnitus ..." (Pierzycki et al., 2015). Here we want to discuss the approach for addressing the question regarding whether oscillatory brain activity may represent a neurophysiological marker of tinnitus with the authors.

First of all, a data driven approach is to be commended but we should not throw away heuristic pathophysiological models of tinnitus, or in Immanuel Kant's words, "Theory without data is empty, data without theory is blind". As history has taught us, with the introduction of the electron microscope in the 1950s, that the reticular theory, as proposed by von Gerlach in 1871 and popularized by Golgi, using his staining method was incorrect. The brain is not one single continuous network as Golgi thought, but the brain is composed of individual cells as Santiago Ramon y Cajal proposed, and that these individual cells are structured in multiple correlated and anticorrelated networks as network science approaches suggest (Bullmore and Sporns, 2009, 2012; Hagmann et al., 2008). If Golgi was correct, the authors' assumption would be very valid but history has favored the Cajal's vision of neuroscience. This has important implications for perception, such as perception of a phantom sound. Taking into account that neuronal mechanisms of conscious perception which are essential in all unconscious states such as in anesthesia, coma, and deep sleep are associated with an absence of tinnitus. Thus, without an awake brain there is no tinnitus percept (De Ridder et al., 2014). All complex 
adaptive systems like the Internet, economy, ant society, or brain share common characteristics; emergence is one of the most important characteristics (Holland, 2014; Johnson, 2010). Emergence is a process whereby larger entities, patterns, and regularities arise through interactions among smaller or simpler entities that themselves do not exhibit such properties (Zalta, 2012). All parts of car do not make a car. Only when all those parts are put together in a very specific way and are (functionally) connected in a very specific way does a functioning car emerge. In a similar way, tinnitus can be considered as an emergent property of a 'tinnitus network' (De Ridder et al., 2014; Schlee et al., 2009), and this implies one has to study tinnitus as a network phenomenon and not just by analyzing activity or power changes. This is in accordance with new concepts of brain disorders as emerging from connectivity changes (Fornito and Bullmore, 2015).

If tinnitus is indeed an emergent property of network activity, it is theoretically conceivable that the network reconfiguration itself, without altered activity, can lead to tinnitus. In this setting whole scalp EEG power can be normal, i.e. the same as in non-tinnitus controls. However, even assuming that tinnitus is associated with mandatory activity changes does not necessarily lead to whole scalp power effects. It is well known that the different restingstate networks in the brain are not all activated simultaneously. Different networks are anti-correlated (Fox et al., 2005) thereby cancelling out each other's network activity, which could also preclude a global whole scalp effect. Furthermore, it is also possible that tinnitus could be related to activity changes in small and focal areas, whose signal will not be powerful enough to change the whole scalp power. This is analogous to what is well known in clinical research. A simple example can demonstrate this point. In general, tinnitus does not respond to anti-seizure medication, as metaanalytic studies have demonstrated (Hoekstra et al., 2011), but a small subgroup of typewriter tinnitus patients (Levine, 2006) does respond very well to anticonvulsants. Response to carbamazepine is even considered a diagnostic criterion. But because this is a rare entity, in studies combining all kinds of tinnitus patients this positive response will not be powerful enough to modify the global negative effect.

Furthermore, there are statistical reasons why it is difficult to understand the authors' approach. Pierzycki and coworkers discuss in detail the findings from cross-sectional group comparisons between tinnitus subjects and controls. If it was the motivation of the authors to address the question whether tinnitus is related to oscillatory changes, they should have chosen a design, which compares "tinnituson" and "tinnitus-off" status using a withinsubjects design. They further mention in the introduction that clinical trials in tinnitus have used EEG power spectra as outcome measures. If they were interested in addressing the usefulness of this approach they should have addressed the relationship between treatment-induced changes in oscillatory brain activity and behavioral changes. However the authors have not completed either of the two mentioned approaches. Instead they performed only testretest measurements (ignoring the individual behavioral changes between the two time points) and correlated whole scalp EEG measures with different psychosocial and psychoacoustic tinnitus variables. Moreover, they used relatively small and clinically inhomogeneous samples for their analysis. Thus, the only conclusion they can draw from their data is, that in the investigated small sample there was no significant correlation between behavioral tinnitus variables and whole scalp EEG power spectra. Moreover, all conclusions from this analysis have to consider the epistemological principle that the absence of evidence for an assumed relationship is methodologically fundamentally different from the evidence of absence of this relationship (Altman and Bland, 1995).

In addition, the authors indicate, "We did use spatial analysis to attenuate artifacts but not a spatial analysis with a source model. Our reason for not doing so was that there were no trends in our results to suggest that spatial analysis would be effective and so post-hoc data exploration cannot be justified" (Pierzycki et al., 2015). However, over the past 25 years source analysis has become a standard, not 
only in biomedical imaging (including EEG), but in countless engineering fields whenever a system of emitters and sensors is involved (Comon and Jutten, 2010). When the sensors record a mixing of the source processes, there always exists a linear combination of the sensor signals providing a signal with higher fidelity with respect to the source process as compared to the signal recorded at any electrode. This simple fact implies that higher statistical power in statistical tests can be achieved at the source level, which invalidates the arguments of the authors. The only case when we can be sure not to miss anything staying at the sensor level is when we use measures that are invariant to linear transformations. This is the case for instance, of the geodesic distance on the Riemannian manifold of positive definite matrices endowed with the Fisher Information metric, which indeed enjoys this property (Bhatia, 2007), but the approach of the authors does not.

In conclusion, we challenge their approach that, "Only if general effects are found at the whole scalp level would it be appropriate to undertake further refined spatial analysis ..." (Pierzycki et al., 2015) and conclusion "... that resting state whole scalp EEG should not be used as a biomarker for tinnitus" (Pierzycki et al., 2015). Based on philosophical, historical, clinical, pathophysiological, and network science in addition to statistical reasons, we disagree with the authors' recommendations to only process EEG data when whole scalp EEG demonstrates a change in power. We suggest instead to use a theoretical model of brain functioning, and combine this with data-driven approaches when possible in order to extract meaningful information from data. To use the authors' own words we would also argue that the claim that, "... greater caution should be exercised in regard to reporting of findings ..." (Pierzycki et al., 2015) is also valid for negative findings.

\section{References}

Altman, D.G., Bland, J.M., 1995. Absence of evidence is not evidence of absence. BMJ 311, 485. Bhatia, R., 2007. Positive Definite Matrices Pricenton. Univserity Press, New Jersey.

Bhatia, R. (2007) Positive Definite matrices, Princeton University press.

Bullmore, E., Sporns, O., 2009. Complex brain networks: graph theoretical analysis of structural and functional systems. Nat. Rev. Neurosci. 10, 186e198.

Bullmore, E., Sporns, O., 2012. The economy of brain network organization. Nat. Rev. Neurosci. 13, $336 \mathrm{e} 349$.

Comon, P., Jutten, C., 2010. Handbook of Blind Source Separation: Independent Component Analysis and Applications. Academic Press, Oxford.

De Ridder, D., Joos, K., Vanneste, S., 2014. The enigma of the tinnitus-free dream state in a Bayesian world. Neural Plast. 2014, 612147.

De Ridder, D., Vanneste, S., Weisz, N., Londero, A., Schlee, W., Elgoyhen, A.B., Langguth, B., 2014 Jul. An integrative model of auditory phantom perception: tinnitus as a unified percept of interacting separable subnetworks. Neurosci. Biobehav. Rev. 44, $16 \mathrm{e} 32$.

Fornito, A., Bullmore, E.T., 2015. Reconciling abnormalities of brain network structure and function in schizophrenia. Curr. Opin. Neurobiol. 30, 44e50.

Fox, M.D., Snyder, A.Z., Vincent, J.L., Corbetta, M., Van Essen, D.C., Raichle, M.E., 2005. The human brain is intrinsically organized into dynamic, anticorrelated functional networks. Proc. Natl. Acad. Sci. U. S. A. $102,9673 e 9678$. 
Hagmann, P., Cammoun, L., Gigandet, X., Meuli, R., Honey, C.J., Wedeen, V.J., Sporns, O., 2008. Mapping the structural core of human cerebral cortex. PLoS Biol. 6, e159.

Hoekstra, C.E., Rynja, S.P., van Zanten, G.A., Rovers, M.M., 2011. Anticonvulsants for tinnitus. Cochrane Database Syst. Rev. CD007960.

Holland, J., 2014. Complexity. Oxford University Press, Oxford. Johnson, N., 2010. Simply Complexity: a Clear Guide to Complexity Theory, second ed. Oneworld Publications, Oxford.

Levine, R.A., 2006. Typewriter tinnitus: a carbamazepine-responsive syndrome related to auditory nerve vascular compression. ORL J. Otorhinolaryngol. Relat. Spec. 68, 43e46 discussion $46 \mathrm{e} 7$.

Pierzycki, R.H., McNamara, A.J., Hoare, D.J., Hall, D.A., 2015. Whole scalp resting state EEG of oscillatory brain activity shows no parametric relationship with psychoacoustic and psychosocial assessment of tinnitus: a repeated measures study. Hear. Res. 331, 101e108.

Schlee, W., Mueller, N., Hartmann, T., Keil, J., Lorenz, I., Weisz, N., 2009. Mapping cortical hubs in tinnitus. BMC Biol. 7, 80.

Zalta, N.E., 2012. Emergent Properties [Online]. Available by the Stanford Encyclopedia of Philosophy. 